\title{
Type 1 Autoimmune Pancreatitis Extending along the Main Pancreatic Duct: IgG4-related Pancreatic Periductitis
}

\author{
Junya Sato ${ }^{1}$, Hiroyuki Matsubayashi ${ }^{1,2}$, Hirotoshi Ishiwatari ${ }^{1}$, Tatsunori Satoh ${ }^{1}$, \\ Junichi Kaneko ${ }^{1}$, Kazuma Ishikawa ${ }^{1}$, Masao Yoshida ${ }^{1}$, Kohei Takizawa ${ }^{1}$, Yohei Yabuuchi ${ }^{1}$, \\ Yoshihiro Kishida ${ }^{1}$, Kenichiro Imai ${ }^{1}$, Kinichi Hotta ${ }^{1}$, Katsuhiko Uesaka ${ }^{3}$, \\ Keiko Sasaki ${ }^{4}$ and Hiroyuki Ono ${ }^{1}$
}

\begin{abstract}
:
We herein report a unique form of autoimmune pancreatitis (AIP) spreading along the main pancreatic duct (MPD). A 70-year-old man was referred for a small lesion at the pancreatic neck, accompanying an adjacent cyst and dilated upstream MPD. Four years earlier, health checkup images had shown a pancreatic cyst but no mass lesion. Endoscopic ultrasonography showed a contrast-enhanced, tumorous lesion, mainly occupying the MPD. With a preoperative diagnosis of ductal neoplasms mainly spreading in the MPD, Whipple's resection was performed. The resected specimens showed MPD periductitis with IgG4-related pathology, indicating type 1 AIP. Clinicians should practice caution concerning the various AIP forms.
\end{abstract}

Key words: autoimmune pancreatitis, IgG4, periductitis, intraductal neoplasms, diagnosis

(Intern Med 60: 739-744, 2021)

(DOI: 10.2169/internalmedicine.5754-20)

\section{Introduction}

Typical images of autoimmune pancreatitis (AIP) show focal or diffuse pancreatic swelling with delayed enhancement and no obvious upstream duct dilation (1-3). However, AIP sometimes appears in atypical clinical images and mimics pancreatic malignancies (4-6). Recent reports have described various forms of AIP, such as that mimicking intraductal papillary mucinous neoplasms (IPMN) (7-9) and that actually accompanying $\operatorname{IPMN}(10,11)$.

We herein report a case of IgG4-related periductitis in the main pancreatic duct (MPD) meeting the diagnostic criteria for type 1 AIP (2), preoperatively suspected of being ductal neoplasms mainly spreading in the MPD.

\section{Case Report}

A 70-year-old man, whose health checkup images had shown a pancreatic cystic lesion 2 years earlier, was referred to the authors' hospital for an apparent pancreatic mass accompanying the lesion and dilation of the upstream MPD. Four years before referral, a cyst had first been found via abdominal ultrasonography (US); it was located at the pancreatic body and was $14 \mathrm{~mm}$ in size at the time. The cyst had remained stable until two years before the referral (Fig. 1). The patient had a drinking history $(350-500 \mathrm{~mL} /$ day of beer for 52 years) but no smoking history. His personal and familial cancer history was unremarkable.

On referral, the patient's blood test was also unremarkable, including serum amylase and tumor markers (carcinoembryonic antigen and cancer antigen 19-9), although he did have an elevated level of HbA1c (6.9\%, normal: 4.6$6.2 \%$ ). Computed tomography (CT) revealed a $10-\mathrm{mm}$ mass at the pancreatic neck that was hypovascular during the arterial phase (20 seconds) but well-enhanced at the portal phase (70 seconds), accompanied by an adjacent unilocular cyst and dilated upstream MPD (Fig. 2a). Magnetic resonance imaging (MRI) revealed the pancreatic mass to have a low-intensity signal on T1-weighted imaging, an

${ }^{1}$ Division of Endoscopy, Shizuoka Cancer Center, Japan, ${ }^{2}$ Division of Genetic Medicine Promotion, Shizuoka Cancer Center, Japan, ${ }^{3}$ Division of Hepato-Biliary-Pancreatic Surgery, Shizuoka Cancer Center, Japan and ${ }^{4}$ Division of Pathology, Shizuoka Cancer Center, Japan Received: July 2, 2020; Accepted: August 17, 2020; Advance Publication by J-STAGE: September 30, 2020 Correspondence to Dr. Hiroyuki Matsubayashi, h.matsubayashi@scchr.jp 
a

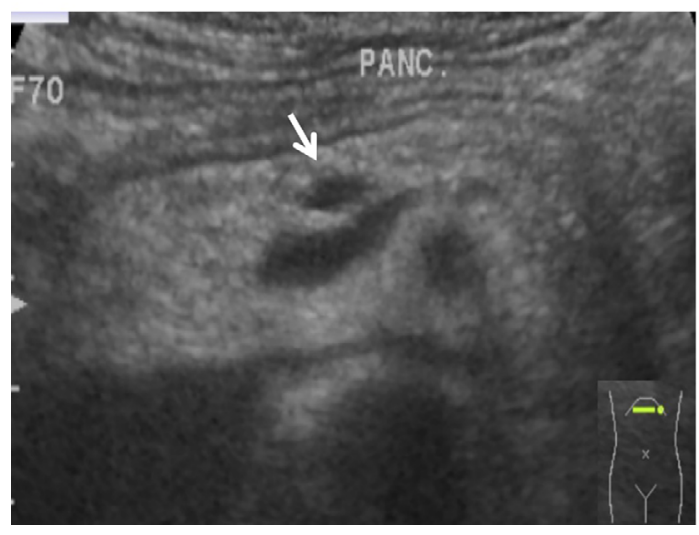

b

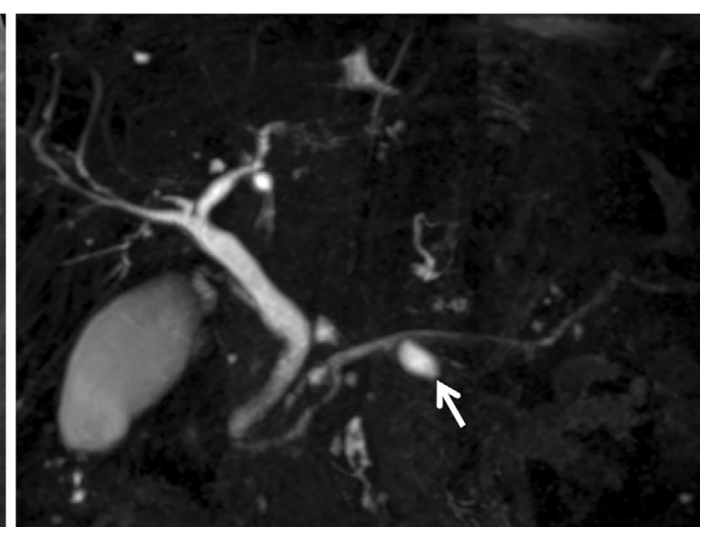

Figure 1. Health checkup images two years before the referral. Abdominal US (a) and MRCP (b) showing a unilocular cyst at the pancreas body without MPD dilation.

a

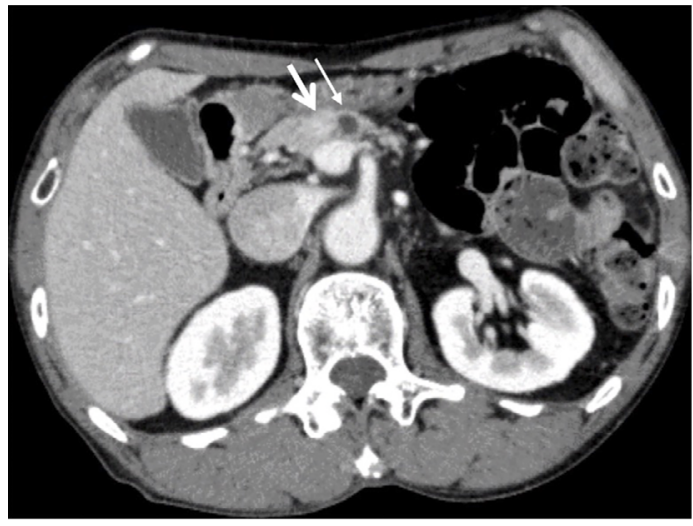

C

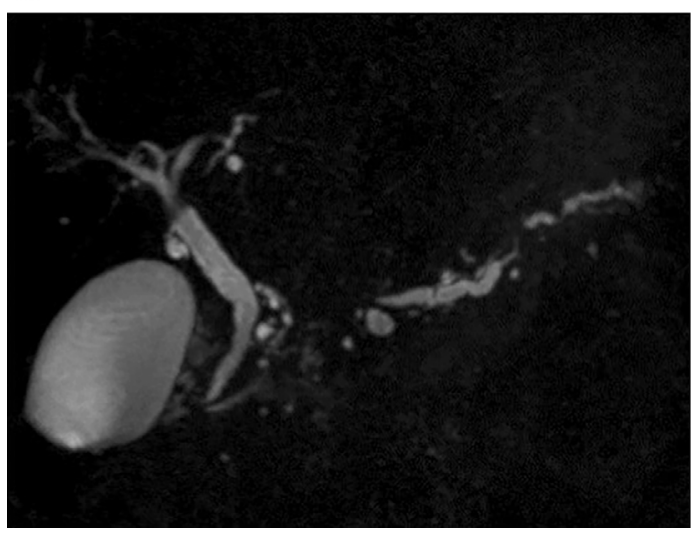

b

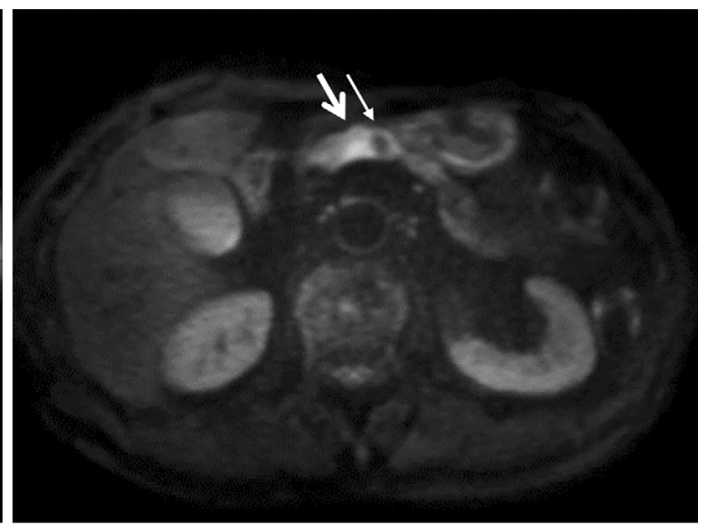

d

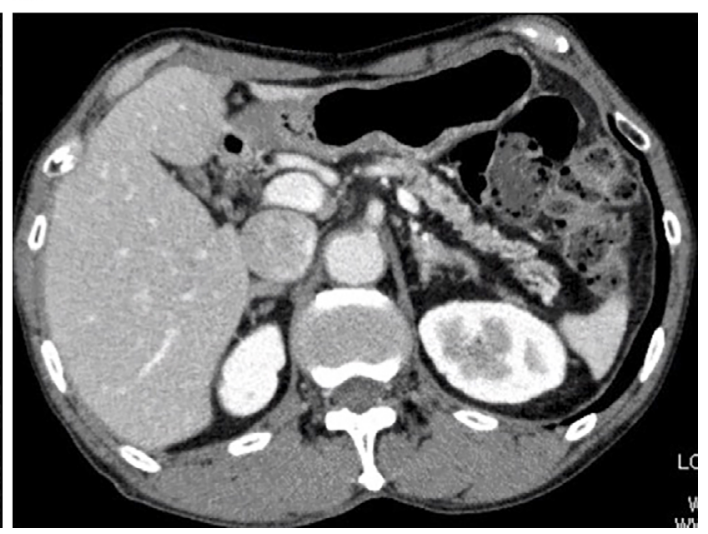

Figure 2. CT (70 s after contrast injection) showing an enhanced mass lesion at the pancreatic neck (arrow) adjacent to the cystic lesion (thin arrow) (a). Diffusion-weighted MRI demonstrating a mass lesion with decreased diffusion capacity and an upstream cyst (b). MRCP demonstrating non-visualized MPD at the pancreatic head and the irregularly dilated upstream duct (c). However, CT demonstrated no mass lesion upstream of the atrophic pancreas (d).

intermediate-intensity signal on T2-weighted imaging, and decreased diffusion capacity (Fig. 2b). Magnetic resonance cholangiopancreatography (MRCP) showed a non-visualized MPD at the pancreatic head and marked dilation of the pancreas body (Fig. 2c). MRCP seemed to show other areas of stricture of the MPD in the pancreas body to tail and also in the biliary hilar portion; however, no mass or stenosis was recognized in these areas by enhanced CT (Fig. 2d). Abdominal US revealed an ill-margined, low-echoic component inside the MPD, which was gradually enhanced by contrastenhanced endoscopic ultrasonography (EUS). Both the upstream and downstream walls of the occupied lesion were 
a

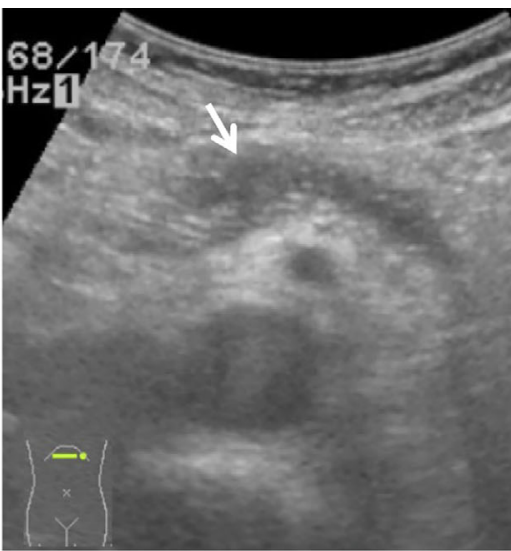

C

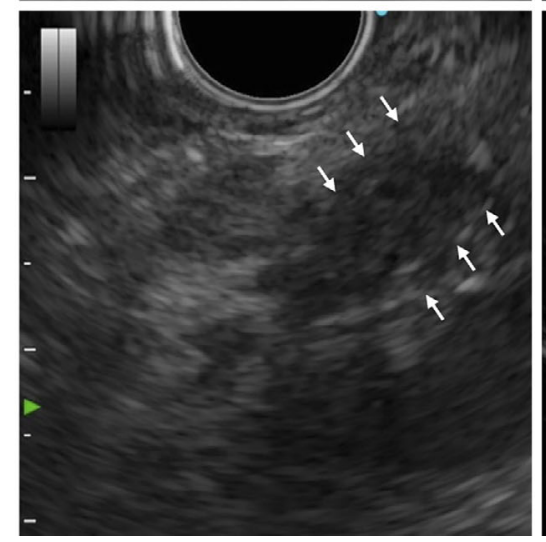

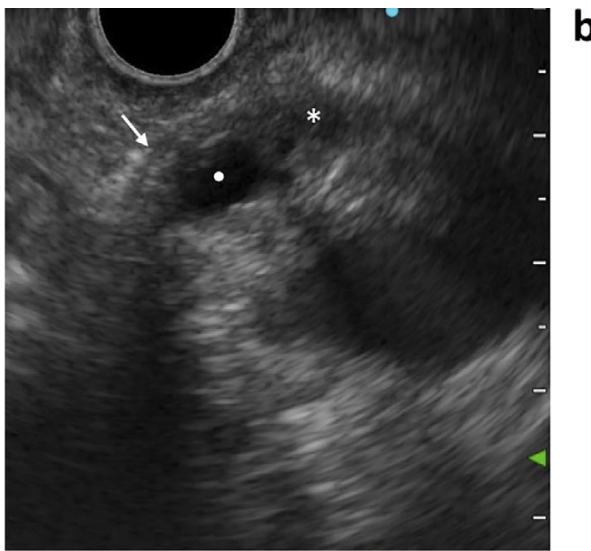

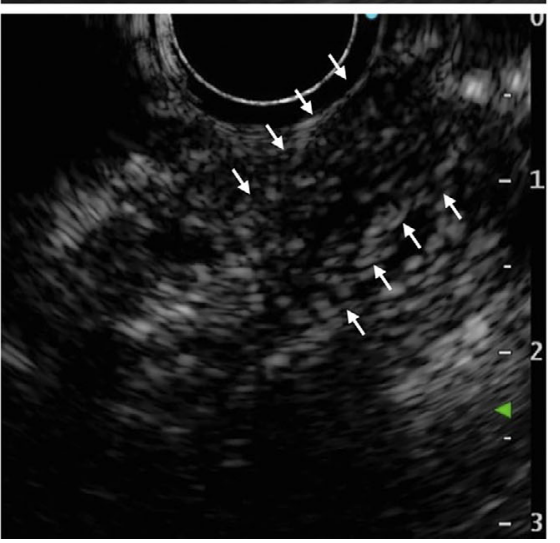

Figure 3. Ultrasonographic views. Horizontal view of abdominal US showing a low-echoic mass lesion in the MPD (a). EUS showing thickened MPD walls (thin arrows) downstream of the mass (asterisk) extending in the MPD (dot) (b) and a low-echoic mass at the pancreas neck inside the MPD (small arrows) (c) with contrast enhancement (d).

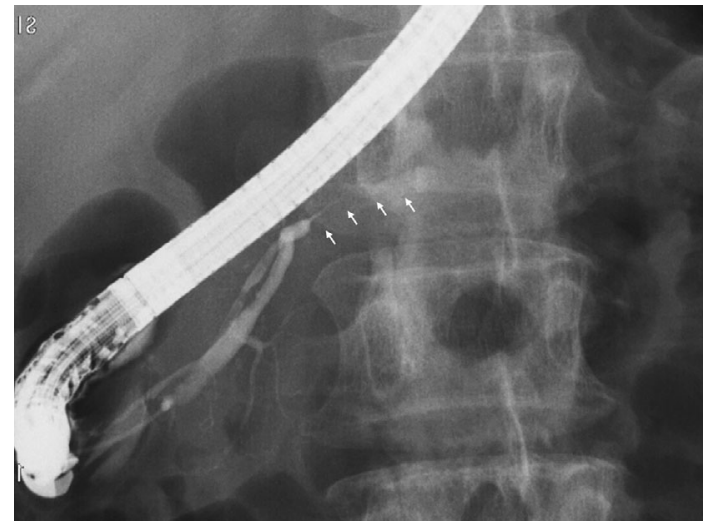

Figure 4. Endoscopic retrograde pancreatography showing MPD stenosis (arrows).

thickened (Fig. 3). Endoscopic retrograde pancreatography had been conducted by the previous hospital and demonstrated stenosis at the corresponding portion of the MPD (Fig. 4). Brushing cytology obtained from the stenotic MPD showed mild cellular atypia but no cancerous cells. Wholetrunk CT and enhanced abdominal MRI did not indicate any lesions suggestive of metastasis but it did show small lesions of interstitial pneumonia. A pancreatic carcinoma mainly in the MPD (possible minimal invasion) was suspected, and pancreatoduodenectomy was performed. The surgical margin was determined by intraoperative ultrasonography and it was also confirmed by aa rapid histological analysis of a frozen section.

Pathology of the resected pancreas showed a whitish mass around the narrowed MPD (Fig. 5). The mass lesion ranged across 5 sections (20 $\mathrm{mm}$ over the pancreatic long axis) around the cyst $(9 \mathrm{~mm})$, and the continuous MPD wall thicknesses toward the head and tail sides were 5 and 10 $\mathrm{mm}$, respectively. On a histological examination, the pancreatic mass demonstrated MPD periductitis at the pancreatic neck composed of abundant lymphoplasmacytic infiltration with numerous IgG4-positive cells (20 cells/high-power field), storiform fibrosis, and obliterative phlebitis around the MPD (Fig. 5), thus satisfying the histological portion of the international consensus diagnostic criteria for type 1 AIP. The cyst was covered with nonneoplastic epithelium $1 \mathrm{~cm}$ in diameter and was recognized alongside the stenotic MPD, suggesting a retention cyst.

One month after surgery, serum IgG4 was first tested and was found to be elevated (262 mg/dL, normal: 11-121 mg/ $\mathrm{dL}$ ). One year after surgery, the patient was in a healthy condition, with no symptoms or evidence of recurrence of the IgG4-related disorder on CT. 
a

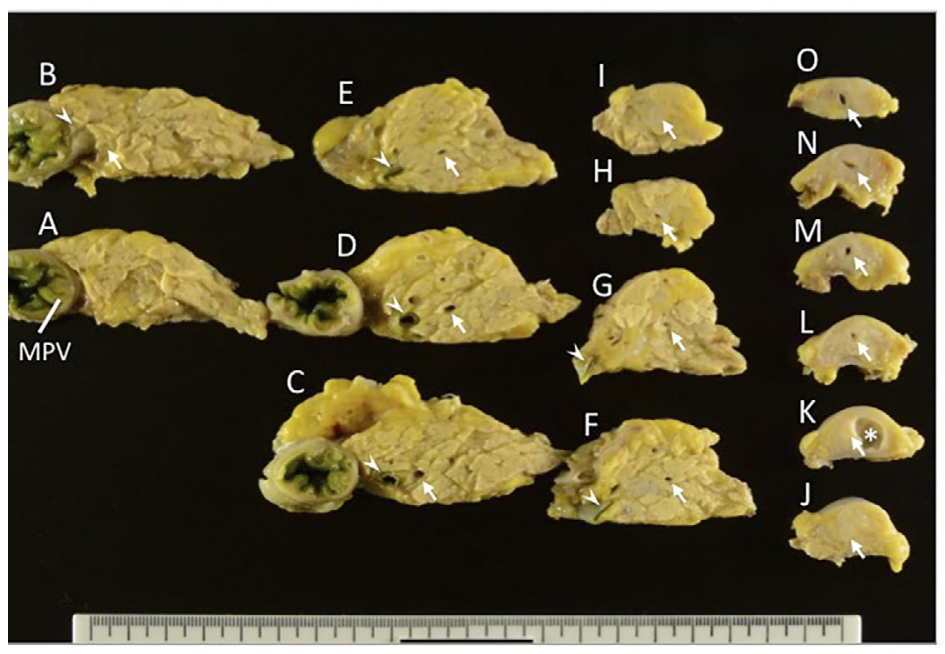

b

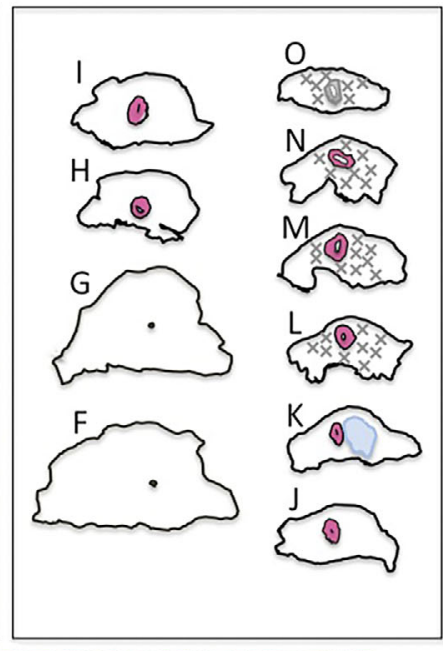

C
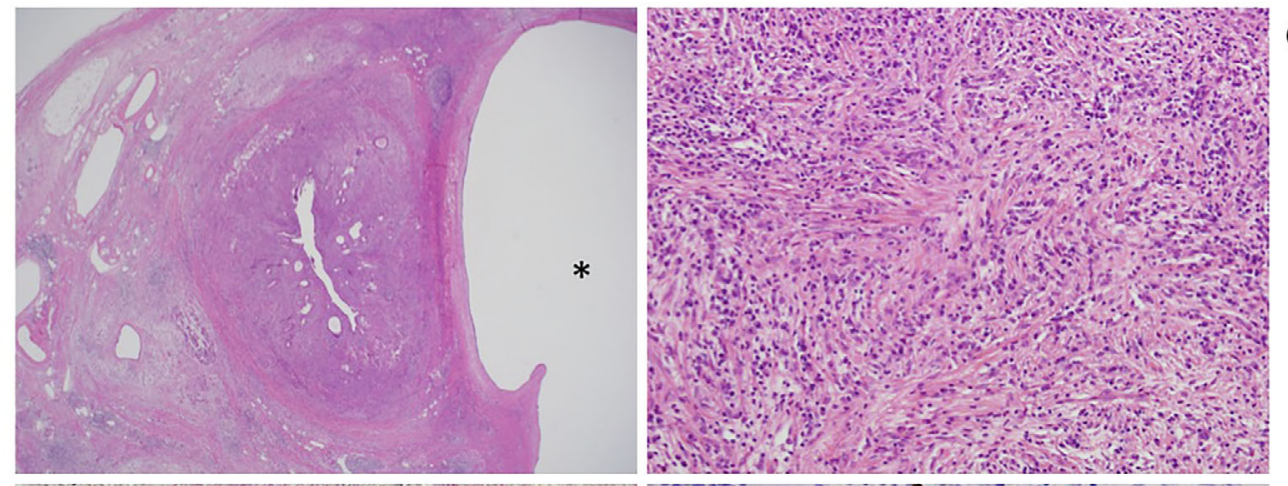

e
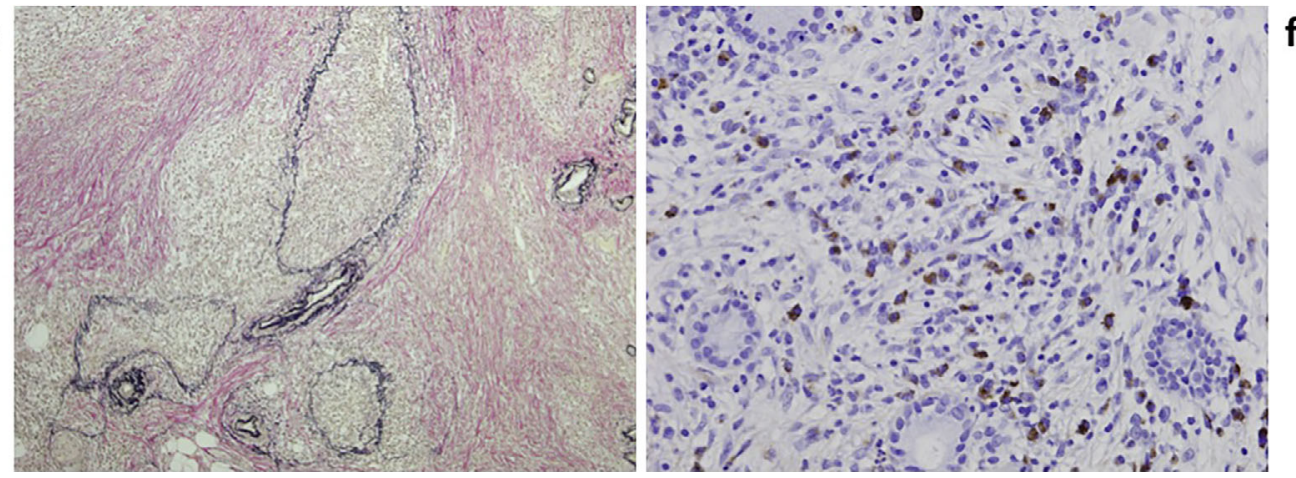

Figure 5. Pathology of the resected pancreas. Macroscopic view of the main lesion. (A to $\mathrm{O}$ are lined up from pancreatic head to body, with arrows indicating the MPD, arrowheads indicating the common bile duct, and an asterisk indicating a pancreatic cyst lesion) (a). A schematic illustration indicating the pathological extent of the IgG4-related lesion marked in pink and the upstream atrophic pancreas with proliferated fibrous tissues marked by crosses $(x)(b)$. Low-powered view of Hematoxylin and Eosin (H\&E) staining showing periductitis with abundant inflammatory cells infiltration around the MPD near the cyst (asterisk) (c). Pathological findings of storiform fibrosis (H\&E staining, $\times 100)(d)$, obliterate phlebitis (Elastica van Gieson, $\times 40)(e)$, and rich IgG4-positive cell infiltration $($ IgG4, $\times 200)(f)$, meeting the criteria for type 1 AIP.

\section{Discussion}

AIP is sometimes difficult to differentiate from pancreatic cancer or lymphoma $(1,3)$. It rarely resembles IPMN (7-9) but is sometimes accompanied by it $(10,11)$. The current case of type 1 AIP demonstrated imaging features different from typical pancreatic cancer or IPMN, showing a slightly enhanced intra-MPD mass with upstream (but no downstream) duct dilation and no mucus secretion.

The imaging findings of the current case suggested several candidates for the presurgical diagnosis. The EUS findings showed the mass component to be mostly within and along the MPD, suggesting curable ductal neoplasms; therefore, a fine-needle aspiration biopsy was not performed due to the risk of seeding (12). Of course, atypical images of 
pancreatic ductal adenocarcinoma or IPMN (less the mucinsecreting type) could not be denied; however, intraductal neoplasms, such as intraductal tubular neoplasm, intraductal tubulopapillary neoplasm $(13,14)$, and acinar cell carcinoma (15), were suspected based on the EUS images. Thus far, cases of AIP mimicking malignant IPMN have been reported, showing nodular lesions protruding inside the MPD (7) or branch duct $(5,9)$, as well as wall thickening around the multilocular cyst (8). The current case added another image variation of type 1 AIP. On reviewing our presurgical examinations, we obtained diagnostic clues concerning type 1 AIP. First, we were able to reexamine ERP for the pancreatic juice cytology by placing endoscopic nasopancreatic duct drainage $(16,17)$ and for a transpapillary biopsy from the stenotic site of the MPD. The risk associated with a pancreatic duct biopsy is thought to be low in cases with an atrophic upstream pancreas (Fig. 2d). Even if serial pancreatic-juice aspiration cytology (SPACE) had given negative results, a forceps biopsy may have shown tissue findings suggestive of IgG4-related pathology. If so, by confirming the elevated serum IgG4 levels and by performing endoscopic ultrasonography-guided fine-needle aspiration (EUS-FNA), the current case of IgG4-related periductitis could have finally been diagnosed and treated with corticosteroid administration $(18,19)$. To avoid unnecessary surgical resection, as in the current case, clinicians need to recall the variety of image patterns of AIPs and measure serum IgG4 when pancreatic lesions show atypical images.

In the present case, type 1 AIP developed during followup monitoring of a pancreatic cyst. The pre-referral images showing only the cyst, two years prior to surgery, suggest that a subclinical, IgG4-related lesion existed but went undetected in the health checkup images. The histology of the current case proved that the non-neoplastic retention cyst was caused by the IgG4-related MPD periductitis. This histology supports the authors' previous study concluding that most cysts associated with AIP are retention cysts, based on how they shrink in response to steroid therapy (20). The problem with pancreatic surveillance is that latent pathological lesions may exist for long periods, undetectable by available imaging modalities.

This case raises another question about the natural course of AIP. Can focal ductitis or IgG4-related small masses $(6,7,9)$ grow to diffuse pancreatitis and further progress to systemic disease, involving other organs? If so, how long does this progression take? In the current case, a mass appeared at least four years after a macroscopically invisible lesion began causing a retention cyst. The authors' previously-reported AIP case (8) demonstrated that wall thickening of the multilocular cyst also appeared after two years of follow-up. Umemura et al. (4) described a case of diffuse-type AIP with a long-term follow-up, involving a multilocular cyst without a mass lesion. In that case, a short narrowing of the MPD was detected 6 months after the initial examination; a 1-cm mass appeared in the 15th month, and diffuse enlargement of the pancreas with a capsule-like rim appeared in the 24th month. The natural course of AIP has not been fully documented, but the speed of change in imaging findings may not differ markedly from that of pancreatic ductal neoplasm (21). Thus, careful differentiation is necessary.

We herein report a case of type 1 AIP, histologically composed of IgG4-related MPD periductitis, mimicking a possibly invasive intraductal neoplasm in presurgical images. Clinicians must be aware of the variety among AIP images and carefully diagnose atypical cases.

Written informed consent was obtained from the patient for permission to publish the present case report.

\section{The authors state that they have no Conflict of Interest (COI).}

\section{References}

1. Okazaki K, Tomiyama T, Mitsuyama T, Sumimoto K, Uchida K. Diagnosis and classification of autoimmune pancreatitis. Autoimmun Rev 13: 451-458, 2014.

2. Shimosegawa T, Chari ST, Frulloni L, et al. International consensus diagnostic criteria for autoimmune pancreatitis: guidelines of the International Association of Pancreatology. Pancreas 40: 352358, 2011.

3. Matsubayashi $\mathrm{H}$, Kakushima N, Takizawa $\mathrm{K}$, et al. Diagnosis of autoimmune pancreatitis. World J Gastroenterol 20: 16559-16569, 2014.

4. Umemura S, Naitoh I, Nakazawa T, et al. Autoimmune pancreatitis presenting a short narrowing of main pancreatic duct with subsequent progression to diffuse pancreatic enlargement over 24 months; natural history of autoimmune pancreatitis. JOP 15: 261265, 2014.

5. Koshita S, Noda Y, Ito K, et al. Branch duct intraductal papillary mucinous neoplasms of the pancreas involving type 1 localized autoimmune pancreatitis with normal serum IgG4 levels successfully diagnosed by endoscopic ultrasound-guided fine-needle aspiration and treated without pancreatic surgery. Intern Med 56: 1163-1167, 2017.

6. Koshita S, Ito K, Fujita N, et al. Localized autoimmune pancreatitis, $9 \mathrm{~mm}$ in size, without strictures of the main pancreatic duct. Gastrointest Endosc 75: 920-922, 2012.

7. Fujie S, Matsubayashi H, Ishiwatari H, et al. Intraductal tubulopapillary epithelial proliferation associated with type 1 autoimmune pancreatitis. J Gastrointestin Liver Dis 27: 83-87, 2018.

8. Kaneko J, Matsubayashi H, Satoh T, et al. Multilocular cyst of type 1 autoimmune pancreatitis masquerading as cancerization of intraductal papillary mucinous neoplasm. Intern Med 59: 199-204, 2020.

9. Nakaji S, Hirata N, Fujii H, et al. A case of focal autoimmune pancreatitis (AIP) mimicking an intraductal papillary mucinous neoplasm (IPMN). Clin J Gastroenterol 6: 329-333, 2013.

10. Naitoh I, Nakazawa $T$, Notohara $K$, et al. Intraductal papillary mucinous neoplasm associated with autoimmune pancreatitis. Pancreas 42: 552-554, 2013.

11. Tabata $T$, Kamisawa $T$, Hara $S$, et al. Intraductal papillary mucinous neoplasm of the pancreas and IgG4-related disease: a coincidental association. Pancreatology 13: 379-383, 2013.

12. Yane K, Kuwatani M, Yoshida M, et al. Non-negligible rate of needle tract seeding after endoscopic ultrasound-guided fine-needle aspiration for patients undergoing distal pancreatectomy for pancreatic cancer. Dig Endosc. Forthcoming.

13. Askan G, Bagci P, Memis B, Basturk O. Intraductal neoplasms of 
the pancreas: an update. Turk Patoloji Derg 33: 87-102, 2017.

14. Basturk O, Adsay V, Askan G, et al. Intraductal tubulopapillary neoplasm of the pancreas: a clinicopathologic and immunohistochemical analysis of 33 cases. Am J Surg Pathol 41: 313-325, 2017.

15. Ban D, Shimada K, Sekine S, et al. Pancreatic ducts as an important route of tumor extension for acinar cell carcinoma of the pancreas. Am J Surg Pathol 34: 1025-1036, 2010.

16. Hanada K, Minami T, Shimizu A, et al. Roles of ERCP in the early diagnosis of pancreatic cancer. Diagnostics (Basel) 9: 90, 2019.

17. Matsubayashi H, Sasaki K, Nagata K, Kanemoto H, Kiuchi R, Ono H. Pancreatic carcinoma mimicking diffuse-type autoimmune pancreatitis: important diagnostic role of pancreatic juice cytology using endoscopic naso-pancreatic drainage. J Dig Dis 13: 287-290, 2012.
18. Matsubayashi H, Imai K, Kusumoto K, Ono H. Suspected autoimmune pancreatitis-an indication for steroid treatment? Dig Liver Dis 42: 525-526, 2010.

19. Kamisawa T, Shimosegawa T, Okazaki K, et al. Standard steroid treatment for autoimmune pancreatitis. Gut 58: 1504-1507, 2009.

20. Matsubayashi $H$, Iwai $T$, Matsui $T$, et al. Pancreatic cystic lesions with atypical steroid response should be carefully managed in cases of autoimmune pancreatitis. J Gastroenterol Hepatol 31: 270-276, 2016.

21. Yachida S, Jones S, Bozic I, et al. Distant metastasis occurs late during the genetic evolution of pancreatic cancer. Nature 467: 1114-1117, 2010.

The Internal Medicine is an Open Access journal distributed under the Creative Commons Attribution-NonCommercial-NoDerivatives 4.0 International License. To view the details of this license, please visit (https://creativecommons.org/licenses/ by-nc-nd/4.0/).

(C) 2021 The Japanese Society of Internal Medicine Intern Med 60: 739-744, 2021 